\title{
U. Rethnam
}

\section{Single incision nailing of the floating knee-do we ignore the knee ligaments?}

Received: 13 December 2005 / Accepted: 13 December 2005 / Published online: 11 April 2006

C) Springer-Verlag 2006

\begin{abstract}
The incidence of knee ligament injuries in the floating knee is as high as $53 \%$ documented in the literature. The single incision technique (antegrade tibial and retrograde femoral nailing through a single incision at the knee) although a good technique in terms of speed and ease, has its own disadvantages. Repair or reconstruction of a torn anterior or posterior cruciate ligament after a single incision technique can be a difficult proposition. Antegrade femoral and tibial nailing (two incisions) makes treatment of knee ligament injuries easier.
\end{abstract}

I read with great interest the article titled "Management of ipsilateral femoral and tibial fractures" (International Orthopaedics Aug 2005;29(4):245-250).

The "single incision" femoral and tibial nailing does have the advantage of being a quick and easy method of fixation of this difficult injury. There is however, a major drawback to this technique which I would like to point out.

This comment refers to the article available at: http://dx.doi. org/10.1007/s00264-005-0661-7.

\footnotetext{
U. Rethnam

Clinical Research Fellow Orthopaedics,

Wrexham Maelor Hospital,

Wrexham, UK

U. Rethnam $(\bowtie)$

18 Bron Y Nant,

Croesnewydd road,

LL13 7TX Wrexham, UK

e-mail: ulfinr@yahoo.com

Tel.: +447779095559
}

It is well documented in the literature that Floating Knee injuries have a higher incidence of knee ligament injuries as compared to isolated femoral or tibial fractures. The incidence of knee ligament injuries can be as high as 53\% according to studies $[1,2]$. The most common injury is an anterior cruciate ligament tear [2] with posterior cruciate ligament, meniscal and collateral ligament injuries following suite. Pre-operative diagnosis of these injuries is difficult as the floating knee injury itself is a "distracting injury" and the inability to perform a proper clinical examination of the knee in the presence of fractures in the same limb.

With the "single incision" technique, repair or reconstruction of a torn anterior or posterior cruciate ligament is a difficult proposition. I feel after an antegrade femoral and tibial nailing of a floating knee, the treatment of knee ligament injuries is easier. After surgical stabilisation of the fractures, the ipsilateral knee is assessed for any evidence of instability under the same anaesthesia. Any instability detected can be dealt with at the same time. Moreover, the authors of the study have obtained results similar to studies where antegrade femoral nailing was done.

Therefore the "single incision" technique for the treatment of the floating knee injury has its own advantages and disadvantages.

\section{References}

1. Paul GR, Sawka MW, Whitelaw GP (1990) Fractures of the ipsilateral femur and tibia: emphasis on intra-articular and soft tissue injury. J Orthop Trauma 4(3):309-314

2. Szalay MJ, Hosking OR, Annear P (1990) Injury of knee ligament associated with ipsilateral femoral shaft fractures and with ipsilateral femoral and tibial shaft fractures. Injury 21(6):398400 